\title{
Clustering Based on Node Density in Heterogeneous Under-Water Sensor Network
}

\author{
Sharad Saxena \\ Prof. Department of Computer Application, BIT, Meerut, UP, India \\ E-mail: sharadzoom@gmail.com \\ Shailendra Mishra \\ Prof. \& Head, CSE, BTKET, Dwarahat, District, Almorah, UK, India \\ E-mail: skmishral@gmail.com \\ Mayank Singh \\ Prof. Computer Science \& Engineering, KIET, Ghaziabad, UP, India \\ E-mail: mayanksingh2005@gmail.com
}

\begin{abstract}
An underwater sensor network comprise of sensors and vehicles to perform numerous tasks. In underwater ad-hoc sensor network acoustic signals are transmitted through multi-hop sequence so as to save sensors' energy and to achieve longer life time. Recharging batteries of deep water deployed sensors is practically not feasible. Clustering is the best strategy to achieve efficient multi-hopping, where cluster head is made responsible to collect local data and forward it to the sink. Cluster-head selection is the challenging job in a cluster, as it loses its energy in transmitting its own data and aggregated data, as compared to other sensors. In this paper we have proposed an Under Water Density Based Clustered Sensor Network (UWDBCSN) scheme using heterogeneous sensors. The scheme utilizes two types of sensors: one having high energy capacity, working as cluster head, having small quantity and other are ordinary sensors in huge quantity. Further cluster-head selection is based on node degree i.e. the density of the sensors in a region. The proposed scheme is found to be more energy efficient helps in extending the life time of underwater sensor networks.
\end{abstract}

Index Terms- Sensor-Network, Node Density, Cluster, Energy

\section{Introduction}

Underwater sensor networks [1] are the special kind of networks deployed in deep oceans, lakes and rivers etc. These networks are scalable, mobile and capable of self-organization. Further they work on propagation of acoustic waves [2]. They have wide range of applications ranging from oceanographic data collection, early warning systems to disasters like tsunamis, oil drilling, ecosystem monitoring etc. The data processing and energy efficient communication is a challenging is sue because of limited power supply to a sensor node. Various research issues from physical layer to application layers have been widely discussed in [3]. Although various protocols for wireless sensor networks have been proposed, yet the typical characteristics of underwater acoustic communication channel like variable delays [4] and limited bandwidth needs updated algorithms, protocols and architecture. The points that differentiate terrestrial sensor network and underwater sensor networks are Hardware Cost, Deployment Method, Data Correlation, Power Consumption, Synchronization and Transmission Delay. The underwater sensor network architecture (see Fig. 1) consists of several trees rooted to the base station. Each tree forwards the data to the sink station using multihop sequences.

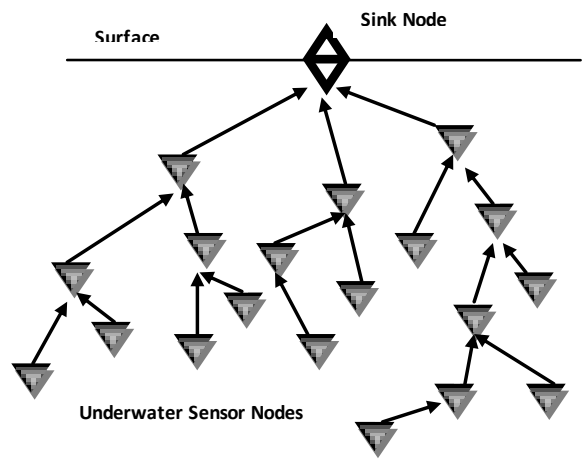

Fig. 1: Underwater Sensor Network Architecture

A multi-hop topology helps in extending the operation of the network but it increases the overhead of intermediate node in processing and forwarding data packets to the neighbor nodes. Therefore nodes near to the sink are overloaded and die earlier. The problem is addressed and taken care by the concept of clustering (see Fig. 2) depicted by several researchers in [5, 6 and 7]. Every cluster has a cluster head that collect local 
data and forward it to parent in hierarchy or to sink node.

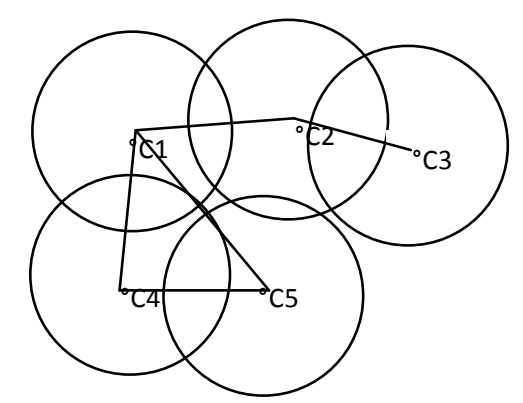

Fig. 2: Clustering in Sensor Networks

Here in this paper we have proposed a new Under Water Density Based Cluster Sensor Network (UWDBCSN) scheme using heterogeneous sensors. Here two types of sensors are used to create clusters. One type having high energy and is working as cluster head and another are normal sensors with average power. The high energy sensors are located in such a way as to cover maximum of low energy sensors in the range and works as cluster-head. If two of high energy sensors are in the range of another the degree of the two sensors are computed to find the network density. The sensor having high node degree is elected as clusterhead and other sensor work as normal node.

The rest of the paper is organized as follows. In section 2 we have shown the related work. Section 3 gives clustering objectives and energy consumption model. Section 4 describes UWDBCSN algorithm. Section 5 shows the result and analys is of the protocol. Finally section 6 concludes the paper.

\section{Related Work}

Authors in [8] discussed clustering protocol based on TDMA/CDMA for network communication. The concept is based on assumption that cluster creation and its maintenance should be based on nodes positions and their movement information obtained from cables and GPS. It makes this approach very restrictive. DUCS protocol [9] is an improvement to this approach. It is used in non-time-critical aquatic monitoring applications in underwater environments. The basic properties of it are: simple, energy aware and GPS-free; it also minimizes the proactive routing exchange, uses data aggregation techniques and does not use flooding. Assuming random node mobility DUCS compensates the high propagation delays of the underwater medium using a continually adjusted timing advance combined with guard time values to minimize data loss. The combination of DUCS with TDMA/CDMA reduces interference and improves communication quality.

Authors in [7] uses a combination of Energy efficient hierarchical clustering and HEED clustering approach to cluster the sensors in the network as to minimize the distance and get maximum utilization of the operational life time of the battery. The self configuring nature of the ad-hoc nodes considered gives the information for creating and regrouping the clusters accordingly. The results in the paper shows that for regrouping the clusters based on the node mobility takes slightly more utilization of power and gives comparatively lesser data transfer for the same amount of time. But without clustering approach battery utilization is comparable more.

Low Energy Adaptive Clustering Hierarchy (LEACH) $[10,12]$ combines the ideas of energyefficient cluster-based routing and media access to achieve good performance in terms of system lifetime, latency, and application-perceived quality. Network consists of clusters in the topology, and each cluster has a cluster head and many cluster member nodes. The cluster head is responsible for the data transmitted between clusters, which can be pre-designed or generated by the election algorithm. LEACH also employed a time division multiple-access (TDMA) MAC protocol developed by Clare et al. [11] to retrench energy. The sensor nodes are allowed to stay in sleep state and keep radios powered-down for a long time period to save power when using a TDMA approach. The cluster head must always be awake to receive all the data from its member nodes and transmit the data to neighbor cluster heads. In Energy Efficient Clustering with Self-organized ID Assignment (EECSIA ) [12], a network first selects the nodes in the high-density areas as cluster heads and then assigns a unique ID to each node based on local information. Further, cluster heads are periodically updated according to the nodes residual energy and density. This method is independent of time synchronization. It does not rely on the nodes geographic locations.

\section{Clustering Objective and Energy Consumption Model}

The proposed method is designed for a densely deployed underwater sensor network. A cluster head consume significantly large amount of energy when transmitting processed data to a remote sink station, therefore the objective is to minimize cluster heads such that each node in the network belongs to a cluster. A node in UWDBCSN may exist in any of the four states: cluster-head, 1-hop neighbor node, 2-hop neighbor node and un-clustered node. Each node can relay its data within 2-hops to the nearest cluster head. A node with high residual energy is elected as cluster head. In UWDBCSN clustering is fully distributed. Each node having high energy interacts with other nodes to form cluster. Thus the scheme is better than centrally controlled schemes as it eliminates central coordination and enables the node to act independently. The set of all possible cluster heads in our scheme is denoted by $C_{h}$. We have considered 2-D under-water network model consisting of $N$ nodes in (X $\mathrm{x} Y$ ) network field (See Fig. 3). 


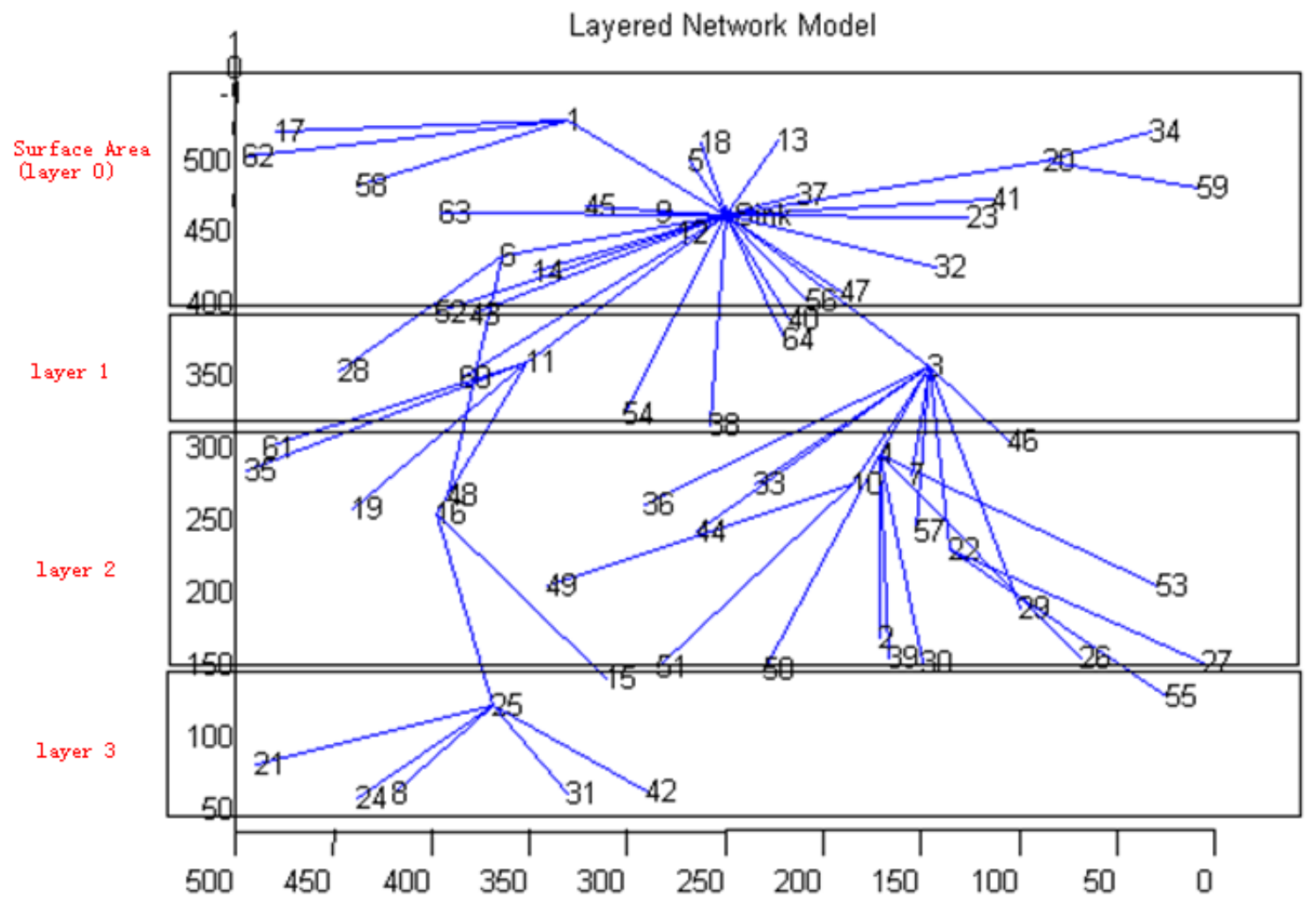

Fig. 3: Network Model

The network model has the following assumptions:

- Sensor nodes are randomly deployed and follow a hierarchical layered approach.

- There is one sink station located at the surface of the water.

- Nodes are not aware of location.

- All nodes have similar processing and communicating range capabilities.

- The network is heterogeneous therefore energy heterogeneity is used.

- Two types of sensor nodes exist: one having comparatively high energy and another of average life time.

- Unique network IDs are used to distinguish high energy sensors versus normal sensors.

We have considered the energy consumption model suggested in [13]. According to which energy is used for modulation $\left(E_{p}\right)$ and for communication $\left(E_{c}\right)$. In our discussion we have considered both free space power loss $\left(\mathrm{d}^{2}\right)$ and mu lti-path fading loss $\left(\mathrm{d}^{4}\right)$ channel models. Where, $d$ is the distance between the transmitter node and receiver node. If $d_{0}$ is the threshold distance depending on the environment then:

$$
\left.\begin{array}{c}
E_{c}=\varepsilon_{\mathrm{fs}} \text { for } \mathrm{d}<\mathrm{d}_{0} \\
E_{c}=\varepsilon_{\mathrm{mp}} \text { for } \mathrm{d}>=\mathrm{d}_{0}
\end{array}\right\}
$$

Thus in order to transmit $k$ bit message to a distance $d$ the radio expends as follows:

$$
E_{t x}(\mathrm{k}, \mathrm{d})=\left\{\begin{array}{l}
\mathrm{k} E_{p}+\mathrm{k} \varepsilon_{\mathrm{fs}} \cdot \mathrm{d}^{2}, \mathrm{~d}<\mathrm{d}_{0} \\
\mathrm{k} E_{p}+\mathrm{k} \varepsilon_{\mathrm{mp}} \cdot \mathrm{d}^{4}, \mathrm{~d}>=\mathrm{d}_{0}
\end{array}\right.
$$

To receive $k$ bit message, the radio expends as follows:

$$
E_{r x}(\mathrm{k})=\mathrm{k} E_{i}
$$

\section{The UWDBCSN Algorithm}

Among $N$ nodes let $N_{p}$ nodes have high energy level and $N_{q}$ are the normal nodes, such that $\mathrm{N}=\mathrm{N}_{\mathrm{p}}+\mathrm{N}_{\mathrm{q}}$. A node in $N_{q}$ is the neighbor of a node in $N_{p}$ if it is in its radio range. Since we have no GPS or other location aware-mechanism available, we use node degree to compare the density of nodes in the network. The node degree $\left(N_{d \text { eg }}\right)$ of a node in $N_{p}$ is the total nu mber of node connected to it.

$$
\begin{gathered}
N_{\text {deg }}(\mathrm{i})=\operatorname{count}\left(N_{q}(\mathrm{j}) \mid \operatorname{dist}(\mathrm{i}, \mathrm{j})\right)<T_{r}, \\
\text { i } \in N_{p} \text { and } \mathrm{j} \in N_{q}
\end{gathered}
$$

Where $\operatorname{dist}(i, j)$ represent the distance between nodes $i$ and $j . T_{r}$ represents the transmitter range of a node and count is number of elements in the finite set. A node in the network belongs to exactly one cluster. The 
minimization of cluster number is equivalent to maximizing average cluster size while maintaining full coverage. The nodes which are not in the radio range of the cluster head, communicates though intermediate nodes using 1-hop or 2-hop sequencing. Clustering process involves four types of messaging $(M)$ :

$M_{\text {state }}:$ indicate current state.

$M_{d e g}$ : indicate node degree.

$M_{\text {join }}$ : un-clustered node to cluster head to join.

$M_{\text {deny }}$ : unwillingness to work as cluster head.

$M_{\text {hello }}$ : hello message.

\subsection{Initial Cluster Creation}

Initially all nodes $N$ are un-clustered. All nodes in $N_{p}$ sends broadcast hello message to the neighbor node in the radio range $T_{r}$ and time interval 0 to $T_{\max }$. After receiving response from nodes in $N_{q}$, nodes in $N_{p}$ calculates node degree. Thereafter every node in $N_{p}$ sends $M_{d e g}$ message to all its neighbor node in $N_{p}$ with

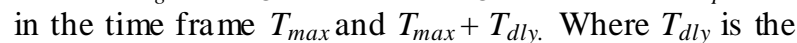
delay of node $i$ given by:

$$
T_{d l y .}=\beta \mathrm{e}^{\wedge}\left(1 / N_{d e g}(\mathrm{i})\right)
$$

$\beta$ is a given constant such that $0<T_{d l y}<=T_{\max }$. If the node degree of all nodes in $N_{p}$ is less than its own, then a random ID is allocated to it as its unique identification number and the node is elected as cluster head. The elected head sends a $M_{\text {state }}$ message to all its neighbor to indicate its state. It is shown in Fig. 4 below.

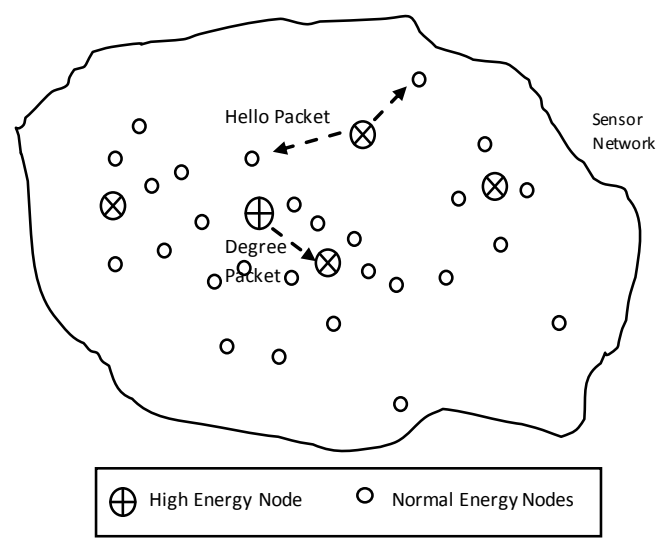

Fig. 4: Message flow in sensor network

From (5) it is evident that node delay time is an increasing function which means a node with large node degree among its neighbors has more probability to be elected as cluster head (see Algorithm 1). Other nodes having degree less than cluster head node behaves like a normal node. A normal node on receiving cluster head state message sends a confirmation message, if it is not a member of any cluster head. Thus a normal node becomes 1-hop member of a cluster head and self generate its unique ID. The normal node sends its state message to its neighbors. The other un-clustered members on receiving message declared them as 2 -hop neighbor and generate their unique ID (see Algorithm 2). The unique IDs for cluster head and other node can be generated by using orthogonal codes. The scheme is shown in Table 1.
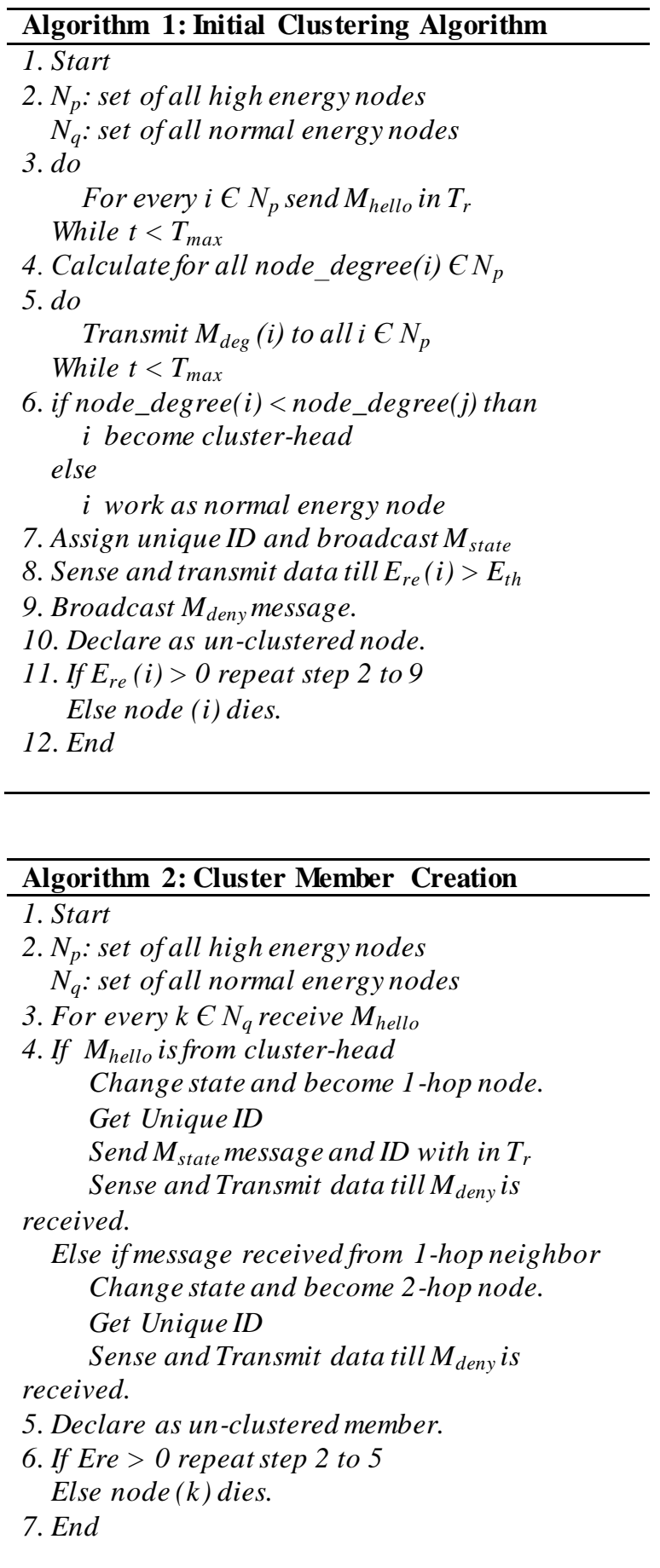
Table 1: ID for cluster head and normal nodes

\begin{tabular}{|c|c|c|}
\hline $\begin{array}{c}\text { Cluster Head ID } \\
\text { (NOVSF Code SF-8) }\end{array}$ & 1-Hop node ID & 2-Hop node ID \\
\hline 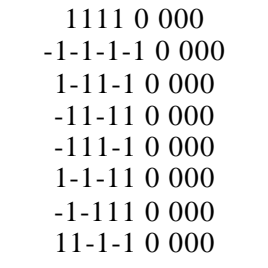 & 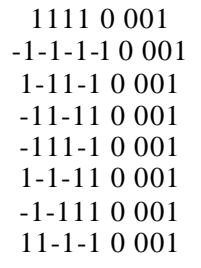 & 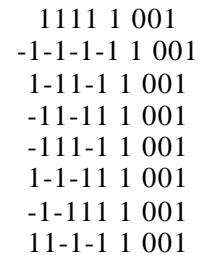 \\
\hline
\end{tabular}

The unique IDs generated in Table 1 are based on Non Orthogonal Variable Spreading Factor based unique code generation technique [14]. Here we have used Spreading Factor (SF)-8 to generate 8 unique codes; however more codes can be generated by increasing spreading factor. The network ID is of $8 \mathrm{~b}$ it long and a cluster head has last four bits set to 0 indicating a cluster head. Nodes at 1-hop neighbor has fifth bit of ID set to 0 and rest of three bits can be used to generate and assign unique code (from 001 to 111) to the other nodes. Similarly, for 2-hop nodes fifth bit is set to 1 and rest of three bits can be used to generate and assign unique code (from 001 to 111) to the other nodes. Hence 1-hop and 2-hop neighbors are identified uniquely.

\subsection{Data Migration and Energy Dissipation in Clustering}

Since, 1-hop neighbor has the same ID as of cluster head except last 4 bits and 2-hop neighbor has the same ID as of its parent 1-hop node except the last 4 bits, there is no need for cluster head to store information regarding nodes belonging other cluster heads. Let, $S 1$ be set of all 1-hop member nodes and $S 2$ is a set of all 2-hop member nodes for a given cluster. When TDMA schedule is executed by each node in the cluster, each node in S2 (represented as S2 (i)) start transmitting sensed data to its parent 1-hop neighbor in its allotted time slot. Each 1-hop node in S1 (represented as S1 (j)) aggregates all received data and send them to the cluster head $C_{h}$. The distance between the nodes is assumed to be less than $d_{0}$. The energy consumption of $\mathrm{S} 1$ (i) and $\mathrm{S} 2(\mathrm{j})$ is as:

$$
\begin{aligned}
\mathrm{E}_{\mathrm{S} 2(\mathrm{j})} & =\mathrm{k}^{*} \mathrm{E}_{\mathrm{p}}+\mathrm{k} \cdot \mathrm{b} * \varepsilon_{\mathrm{fs}} * \operatorname{dist}^{2}(\mathrm{~S} 2(\mathrm{i}), \mathrm{S} 2(\mathrm{j})) \\
\mathrm{E}_{\mathrm{S} 1(\mathrm{i})}= & \mathrm{k}^{*}\left[\mathrm{E}_{\mathrm{p}} * \operatorname{count}(\mathrm{A} 1-\operatorname{hop}(\mathrm{S} 1(\mathrm{i}))\right. \\
& +\mathrm{E}_{\mathrm{agg}} * \operatorname{count}(\mathrm{A} 2-\operatorname{hopS} 1(\mathrm{i}))+\mathrm{E}_{\mathrm{p}} \\
& \left.+\varepsilon_{\mathrm{fs}} * \operatorname{dist}^{2}\left(\mathrm{~S} 1(\mathrm{i}), \mathrm{C}_{\mathrm{h}}(\mathrm{g})\right)\right]
\end{aligned}
$$

Where $b$ is the aggregated data, $k$ is length of data transmitted in single transmission, (A1 - hop (S1 (i)) denotes the set of all me mbers in S1 (i), (A2 - hopS1(i)) denotes the set of all 1-hop members of each cluster head $C_{h}(g)$. Finally each cluster head aggregates the data and forward it to the fixed base station. If the distance of base station is greater than $d_{0}$ the multipath model is used for transmission shown in (8) otherwise free space model shown in (9) is used for communication. The energy dissipated in cluster head $C_{h}(g)$ is:

$$
\begin{aligned}
\mathrm{EC}_{\mathrm{h}}(\mathrm{g})= & \mathrm{k} *\left[\mathrm{E}_{\mathrm{p}} * \operatorname{count}(\mathrm{A} 1-\operatorname{hop}(\mathrm{S} 1(\mathrm{i}))\right. \\
& +\left(\mathrm{E}_{\mathrm{agg}} * \operatorname{count}(\mathrm{A} 2-\operatorname{hop} \mathrm{S} 1(\mathrm{i}))+1\right)+\mathrm{E}_{\mathrm{p}} \\
& \left.+\varepsilon_{\mathrm{mp}} * \operatorname{dist}^{4}\left(\mathrm{C}_{\mathrm{h}}(\mathrm{g}), \sin k\right)\right] \\
\mathrm{EC}_{\mathrm{h}}(\mathrm{g})= & \mathrm{k} *\left[\mathrm{E}_{\mathrm{p}} * \operatorname{count}(\mathrm{A} 1-\operatorname{hop}(\mathrm{S} 1(\mathrm{i}))\right. \\
& +\left(\mathrm{E}_{\mathrm{agg}} * \operatorname{count}(\mathrm{A} 2-\operatorname{hop} \mathrm{S} 1(\mathrm{i}))+1\right)+\mathrm{E}_{\mathrm{p}} \\
& \left.+\varepsilon_{\mathrm{mp}} * \operatorname{dist}^{2}\left(\mathrm{C}_{\mathrm{h}}(\mathrm{g}), \sin k\right)\right]
\end{aligned}
$$

Where $\left(\mathrm{C}_{\mathrm{h}}(\mathrm{g})\right.$, sink) is the distance between cluster head $g$ and sink node. If $E_{t h}$ be the energy threshold for each cluster head to decide whether to continue to serve as a cluster head in the next round. If the residual energy, $E_{r e}$ of the current cluster head is below $E_{t h}$. It will be replaced by one of its neighbor node having node degree maximum among its neighbor. The previous cluster node now behaves as normal node in the next round.

\section{Results and Analysis}

We have simulated the wireless sensor networks in MATLAB environment in 500x500 fields. Table 2 shows the basic simulation parameters used. For analysis and result comparison we have compared UWDBCSN protocol with DUCS, Leach and EECSIA protocol in terms of network lifetime, network sustainability and performance of availability of high energy nodes over normal nodes. The results are shown in Fig. 7, Fig. 8 and Fig. 9 respectively.

Table 2: Simulation parameters

\begin{tabular}{|l|l|}
\hline \multicolumn{1}{|c|}{ Parameter } & \multicolumn{1}{c|}{ Value } \\
\hline Network size & $500 \times 500$ \\
\hline Sensor nodes & 150 \\
\hline $\mathrm{E}_{\mathrm{th}}$ & $1 \mathrm{~J}$ \\
\hline $\mathrm{d}_{0}$ & $80 \mathrm{~m}$ \\
\hline Initial energy of High energy sensor & $4 \mathrm{j}$ \\
\hline Initial energy of normal sensor & $2 \mathrm{j}$ \\
\hline$\varepsilon_{\mathrm{fs}}$ & $10 \mathrm{pj} / \mathrm{bit} / \mathrm{m}^{2}$ \\
\hline$\varepsilon_{\mathrm{mp}}$ & $0.0013 \mathrm{pj} / \mathrm{bit} / \mathrm{m}^{4}$ \\
\hline $\mathrm{Message} \mathrm{size}$ & $2000 \mathrm{bits}$ \\
\hline $\mathrm{T}_{\mathrm{r}}$ & $20 \mathrm{~m}$ \\
\hline $\mathrm{T}_{\max }$ & $300 \mathrm{~ms}$ \\
\hline
\end{tabular}


Network life time prediction is based on the time interval between the times, network start its operation and the time until death of the last alive node. We have determined the performance of three protocols over number of rounds. The result is shown in Fig. 7. In UWDBCSN the lifetime is found to be more as compared to others. Fig. 8 is the result obtained when the high energy sensor availability is varied and the effect is observed on the availability of normal nodes in the network. The experiment is repeated for 250 rounds. After a certain value the availability of number of normal node decreases due to some threshold value.

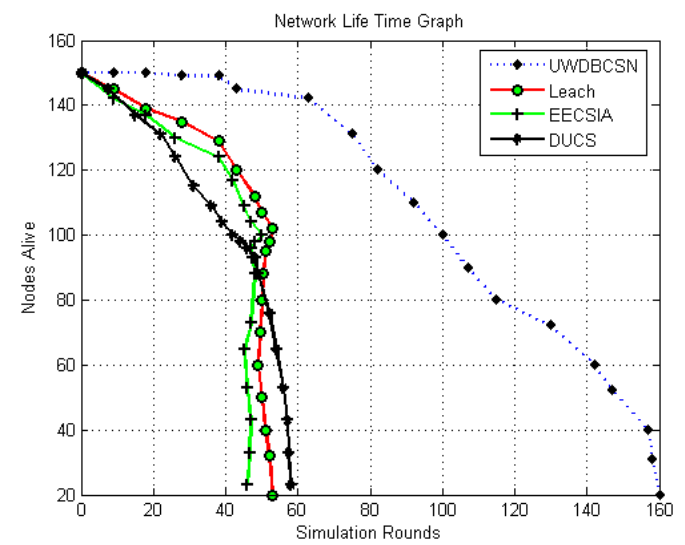

Fig. 7: Network Life Time Graph

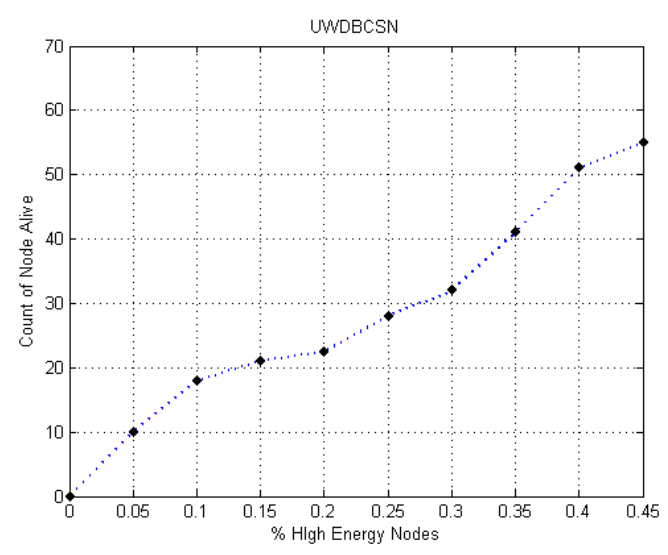

Fig. 8: Network Sustainability

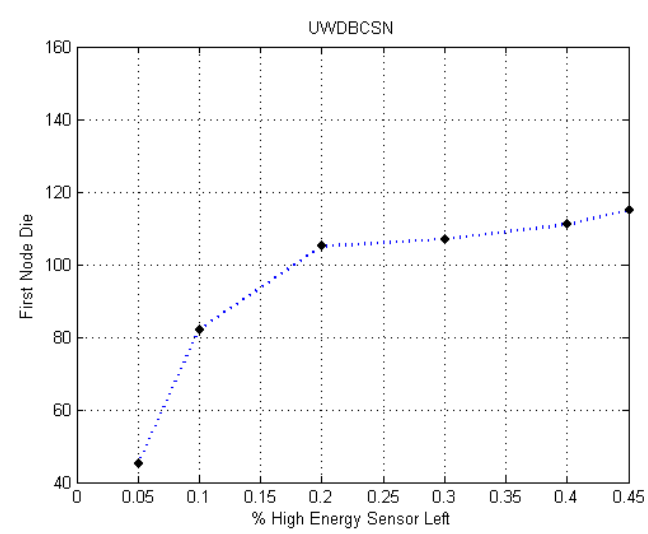

Fig. 9: High energy sensor left till first node die
Network sustainability is measured on the time period during which a network start its operation and continues till the very first node of the network dies. This region is also refereed as stable region. Here the high energy nodes are having twice the energy of the normal node. The first node is observed to die after 43 rounds as shown in Fig. 9. The second node at 82 third is on 102 and soon.

\section{Conclusion and Future Work}

This paper presents UWDBCSN protocol for a distributed environment. The approach is based on clustering concept that explores the density of the sensor nodes to elect cluster-head. The method makes utilization of heterogeneous sensor devices that have diffe rent energy capacity. The protocol help in reducing overall communication cost in electing cluster-head hence increases overall network lifetime. Our simulation results show that the proposed protocol performs better than the other protocol of the family. As a future work we will study the effect of temperature, pressure and stream effect in underwater communication.

\section{References}

[1] J.-H. Cui, J. Kong, M. Gerla and S. Zhou, "Challenges: Building Scalable Mobile Underwater W ireless Sensor Networks for Aquatic Applications", Special issue of IEEE Network on Wireless Sensor Networking, May 2006.

[2] M.Stojanovic, "Acoustic (Underwater) Communications", entry in Encyclopedia of Telecommunications, John G. Proakis, Ed., John Wiley \& Sons, 2003.

[3] J. Heidemann, Y. Li, A. Syed, J Wills and W. Ye, "Research Challenges and Applications for Underwater Sensor Networking", Proceedings of the IEEE Wireless Communications and Networking Conference (WCNC2006), April 3-6, 2006, Las Vegas, Nevada, USA.

[4] J.G. Proakis, E.M. Sozer, J.A. Rice, M. Stojanovic, Shallow water acoustic networks, IEEE Communications Magazine 39 (11) (2001) 114119.

[5] Raja Jurdak, Cristina Videira Lopes, Pierre Baldi, "Battery lifetime estimation and optimization for underwater sensor networks", a john wiley \& sons, inc., publication

[6] Kemal Akkaya* and Andrew Newell "SelfDeployment of Sensors for Maximized Coverage in Underwater Acoustic Sensor Networks", Southern Illinois University Carbondale, IL 62901, submitted to Elsevier, January 13, 2009. 
[7] Andhe Dharani, Member, IAENG, Vijayalakshmi M. N, Vijay Singh, Sumithra Devi K. A, "Power optimization in Ad hoc Sensor Networks using Clustering Approach", Proceedings of the World Congress on Engineering 2011 Vol II, W CE 2011, July 6 - 8, 2011, London, U.K.

[8] F. Salva-Garau and M. Stojanovic, "Multi-Cluster Protocol for Ad Hoc Mobile Underwater Acoustic Networks", In IEEE OCEANS'03 Conference, 2003.

[9] Mari Carmen Domingo,1 Rui Prior. "A DISTRIBUTED CLUSTERING SCHEME FOR UNDERWATER WIRELESS SENSOR NETWORKS", The 18th Annual IEEE International Sy mposium on Personal, Indoor and Mobile Radio Communications (PIMRC'07)

[10] Park,M.K., Rodoplu, V.: UWAN-MAC: An energy-efficient MAC protocol for underwater acoustic wireless sensor networks. IEEE J. Ocean. Eng. 32(3), 710-720 (2007)

[11] Clare, L., Pottie, G., Agre, J.: Self-organizing distributed sensor networks. In: Proc. SPIE Conf. Unattended Ground Sensor Technologies and Applications, vol. 3713, pp. 229-237, Orlando, FL, Apr. 1999.

[12] Qingchao Zheng, Z. Liu, Liang Xue, Yusong Tan, Dan Chen, and Xinping Guan, "An Energy Efficient Clustering Scheme with Self-organized ID Assignment for Wireless Sensor Networks". 2010 16th IEEE International Conference on Parallel and Distributed Systems, 2010.

[13] A. P. Chandrakasan, A. C. Smith and W. B. Heinzelman, "An Application Specific Protocol Architecture for Wireless Microsensor Networks," IEEE Transactions on Wireless Communication, vol. 1, no. 4, 2004, pp 660-669.

[14] Kiran Vadde and Hasan Cam, 2004 A Code Assignment Algorithm for Non-blocking OVSF Codes in WCDMA, Telecommunication Systems 25:3,4, 417-431, Kluwer Academic Publisher, Netherland.

\section{Authors' Profiles}

Sharad Saxena, did his Ph. D. (Computer Science and Engineering) from Uttarakhand Technical University, Dehradun, India in 2012, and received M. Tech. (Computer Science and Engineering) in 2009. He has more than 12 international research publications, and guided $2 \mathrm{M}$. Tech. dissertations. He is member of reviewer committee in journal of repute. His research interest includes Wireless Sensor Networks, Distributed Systems, and Wireless communication, with a focus on Mobile Ad-Hoc Networks.
Shailendra Mishra, did his Ph. D. in 2007 and Master of Engineering Degree (ME) in Computer Science \& Engineering (Specialization: Software Engineering) from Motilal Nehru National Institute of Technology (MNNIT), Allahabad, India in 2000.

His recent research has been in the field of Mobile Computing \& Communication and Advance Network Architecture. He has also been conducting research on Communication System \& Computer Networks with Performance evaluation and design of Multiple Access Protocol for Mobile Communication Network.

Mayank Singh, done his M. E in software engineering from Thapar University and $\mathrm{Ph}$. D. from Uttarakhand Technical University. His Research areas are Software Engineering, Software Testing, Wireless Sensor Networks and Data Mining.

How to cite this paper: Sharad Saxena, Shailendra Mishra, Mayank Singh,"Clustering Based on Node Density in Heterogeneous Under-Water Sensor Network", International Journal of Information Technology and Computer Science(IJITCS), vol.5, no.7, pp.49-55, 2013. DOI: 10.5815/ijitcs.2013.07.06 